\title{
Note on restoring manifest rotational symmetry in hyperfine and fine structure in light-front QED.
}

\author{
Martina Brisudová and Robert Perry \\ Department of Physics \\ The Ohio State University, Columbus, OH 43210.
}

September 21, 2018

\begin{abstract}
We study the part of the renormalized, cutoff QED light-front Hamiltonian that does not change particle number. The Hamiltonian contains interactions that must be treated in second-order bound state perturbation theory to obtain hyperfine structure. We show that a simple unitary transformation leads directly to the familiar Breit-Fermi spin-spin and tensor interactions, which can be treated in degenerate first-order bound-state perturbation theory, thus simplifying analytic light-front QED calculations. To the order in
\end{abstract}


momenta we need to consider, this transformation is equivalent to a Melosh rotation. We also study how the similarity transformation affects spin-orbit interactions. 


\section{Introduction}

Light-front Hamiltonian field theory is being developed as a tool for solving bound state problems in QCD [1, 2]. Cutoffs are introduced that can be lowered using a similarity renormalization group [3], and renormalization can be completed either using coupling coherence [4] or by fixing counterterms to repair symmetries violated by the cutoffs. In the simplest procedure the renormalized, cutoff Hamiltonians are computed perturbatively and may then be diagonalized non-perturbatively to obtain low-lying bound states. Each stage has an approximation scheme associated with it: in the first step, the effective Hamiltonian is calculated to a given order in perturbation theory; and in the second step the effective Hamiltonian is divided into a dominant part, which defines the starting bound-state wave functions, and a perturbation, which is treated to a given order using bound-state perturbation theory. Interactions that change particle number are treated perturbatively. As a consequence, different Fock states decouple and one is left with few-body problems in the leading order. It is therefore important to know to what extent bound states are accurately described by the effective interactions that do not change particle number. A principal signature of the truncation errors in these schemes is a violation of rotational invariance, which is a dynamical symmetry in light-front field theory.

There are two reasons why rotational symmetry is complicated in this approach. The first is that we have formulated the theory on the light front. Rotations are dynamical, and light-front spinors depend on the choice of z-axis. In the weak-coupling limit, however, one expects that rotations should become simple because both boost and rotational 
symmetries are kinematic in the nonrelativistic limit. The second source of complexity is the regularization and renormalization scheme that we use [1, 2]. This has profound effects in QCD [5], but in QED, to obtain the leading interactions cutoffs can effectively be removed. Therefore, to disentangle the two problems it is useful to study QED in the nonrelativistic limit.

Jones et al [6] have studied the ground state hyperfine structure of positronium in this approach. To second order in the coupling there are new interactions between electron and positron that arise from eliminating matrix elements of the Hamiltonian involving highenergy photon emission and absorption. These new interactions have spin-independent as well as spin-dependent parts. The spin-independent interaction, combined with the instantaneous exchange interaction, leads to the Coulomb interaction, and the leadingorder problem reduces to the familiar equal-time Schrödinger equation [2].

The light-front spin-dependent interactions appear to be different from the spindependent interactions found in an equal-time formulation, even in the nonrelativistic limit. For example, if one calculates the hyperfine splitting in the positronium ground state, first-order bound-state perturbation theory gives incorrect results. The triplet state is not degenerate, and the energy of the singlet state is incorrect. This is because the effective Hamiltonian contains a term that does not give a contribution in first-order boundstate perturbation theory, but its contribution in second-order bound-state perturbation theory is of the same order in $\alpha$ as the terms contributing in the first order, $\mathcal{O}\left(\alpha^{4}\right)$. Jones et al [6] effectively summed the second-order bound-state perturbation theory analytically, and showed that it leads to the correct hyperfine splitting in the ground state of 
positronium. Kaluza and Pirner encountered the same problem [7], and they completed the sum numerically.

We propose an alternative approach. We find a simple unitary transformation of the Hamiltonian that alters the problematic term so that it enters at first-order in bound-state perturbation theory. We find the transformation order-by-order in powers of momenta. In order to restore the hyperfine splitting, we need to find the unitary transformation only to the next-to-leading order. A unitary transformation does not change the eigenvalues, and the transformation we obtain makes the calculation much easier. It turns out that the unitary transformation to this order is an expansion of the so called Melosh transformation [8] to next-to-leading order in powers of momenta. The simple formalism enables us to study how the similarity transformation affects the spin-dependent structure of the effective Hamiltonian. This issue is addressed in the third section. The last section contains our conclusions.

\section{Spin-spin interaction in QED}

The effective Hamiltonian for a fermion and an antifermion generated by the similarity transformation [3] using coupling coherence [4] is

$$
H_{\text {eff }}=H_{\text {free }}+V_{1}+V_{2}+V_{2} \text { eff },
$$

where $H_{\text {free }}$ is the kinetic energy, $V_{1}$ is $\mathcal{O}(g)$ emission and absorption, $V_{2}$ is the $\mathcal{O}\left(g^{2}\right)$ instantaneous exchange interaction, and $V_{2}$ eff includes the $\mathcal{O}\left(g^{2}\right)$ effective interactions. 
For simplicity, we only consider the case where the fermion and antifermion have equal mass.

Kinetic energy is diagonal in momentum space, and matrix elements of the interactions are nonzero only between states with energy difference smaller than $\frac{\Lambda^{2}}{\mathcal{P}^{+}}$, which is reflected by an overall cutoff function in the equations below. If the cutoff is chosen within certain limits, the cutoff functions can be approximated by 1 to leading order in $\alpha$ [2].

Matrix elements of the Hamiltonian in a state containing a fermion and antifermion pair are as follows. The kinetic energy is diagonal in momentum space:

$$
\frac{p^{\perp 2}+m^{2}}{p^{+}}+\frac{k^{\perp 2}+m^{2}}{k^{+}}
$$

The emission and absorption of a photon enters at second order.

Let $p_{i}, k_{i}$ be the light-front three-momenta carried by the fermion and antifermion; $\sigma_{i}, \lambda_{i}$ are their light-front helicities; $u(p, \sigma), v(k, \lambda)$ are their spinors; index $i=1,2$ refers to the initial and final states, respectively. The instantaneous exchange interaction mixes states of different momenta:

$$
\begin{gathered}
-g^{2} \bar{u}\left(p_{2}, \sigma_{2}\right) \gamma^{\mu} u\left(p_{1}, \sigma_{1}\right) \bar{v}\left(k_{1}, \lambda_{1}\right) \gamma^{\nu} v\left(k_{2}, \lambda_{2}\right) \\
\times \frac{1}{{q^{+2}}^{2}} \eta_{\mu} \eta_{\nu} \theta\left(\frac{\Lambda^{2}}{\mathcal{P}^{+}}-\left|\left(p_{1}^{-}+k_{1}^{-}\right)-\left(p_{2}^{-}+k_{2}^{-}\right)\right|\right),
\end{gathered}
$$

and so do the effective interactions generated by the similarity transformation:

$$
\begin{gathered}
-g^{2} \bar{u}\left(p_{2}, \sigma_{2}\right) \gamma^{\mu} u\left(p_{1}, \sigma_{1}\right) \bar{v}\left(k_{1}, \lambda_{1}\right) \gamma^{\nu} v\left(k_{2}, \lambda_{2}\right) \\
\times \frac{1}{q^{+}} D_{\mu \nu}(q)\left(\frac{\theta\left(\left|D_{1}\right|-\frac{\Lambda^{2}}{\mathcal{P}^{+}}\right) \theta\left(\left|D_{1}\right|-\left|D_{2}\right|\right)}{D_{1}}+\frac{\theta\left(\left|D_{2}\right|-\frac{\Lambda^{2}}{\mathcal{P}^{+}}\right) \theta\left(\left|D_{2}\right|-\left|D_{1}\right|\right)}{D_{2}}\right) \\
\times \theta\left(\frac{\Lambda^{2}}{\mathcal{P}^{+}}-\left|\left(p_{1}^{-}+k_{1}^{-}\right)-\left(p_{2}^{-}+k_{2}^{-}\right)\right|\right) .
\end{gathered}
$$


where $D_{\mu \nu}(q)=\frac{q^{\perp^{2}}}{{q^{+2}}^{2}} \eta_{\mu} \eta_{\nu}+\frac{1}{q^{+}}\left(\eta_{\mu} q_{\nu}^{\perp}+\eta_{\nu} q^{\perp}{ }_{\mu}\right)-g_{\mu \nu}^{\perp}$ is the photon propagator in lightfront gauge, $\eta_{\mu}=\left(0, \eta_{+}=1,0,0\right) ; q=p_{1}-p_{2}$ is the exchanged momentum, with $q^{-}=\frac{q^{\perp^{2}}}{q^{+}}$; $D_{1}, D_{2}$ are energy denominators: $D_{1}=p_{1}^{-}-p_{2}^{-}-q^{-}$and $D_{2}=k_{2}^{-}-k_{1}^{-}-q^{-}$. It is convenient to add (2) and (3) together, leading to:

$$
\begin{aligned}
& g^{2} \bar{u}\left(p_{2}, \sigma_{2}\right) \gamma^{\mu} u\left(p_{1}, \sigma_{1}\right) \bar{v}\left(k_{2}, \lambda_{2}\right) \gamma^{\nu} v\left(k_{1}, \lambda_{1}\right) \\
& \quad \times \quad\left[g_{\mu \nu}\left(\frac{\theta\left(\left|D_{1}\right|-\frac{\Lambda^{2}}{\mathcal{P}^{+}}\right) \theta\left(\left|D_{1}\right|-\left|D_{2}\right|\right)}{q^{+} D_{1}}+\frac{\theta\left(\left|D_{2}\right|-\frac{\Lambda^{2}}{\mathcal{P}^{+}}\right) \theta\left(\left|D_{2}\right|-\left|D_{1}\right|\right)}{q^{+} D_{2}}\right)\right. \\
& \left.\quad-\frac{\eta_{\mu} \eta_{\nu}}{2 q^{+2}}\left(1-\frac{\theta\left(\left|D_{1}\right|-\frac{\Lambda^{2}}{\mathcal{P}^{+}}\right) \theta\left(\left|D_{1}\right|-\left|D_{2}\right|\right) D_{2}}{D_{1}}-\frac{\theta\left(\left|D_{2}\right|-\frac{\Lambda^{2}}{\mathcal{P}^{+}}\right) \theta\left(\left|D_{2}\right|-\left|D_{1}\right|\right) D_{1}}{D_{2}}\right)\right] \\
& \quad \times \quad \theta\left(\frac{\Lambda^{2}}{\mathcal{P}^{+}}-\left|\left(p_{1}^{-}+k_{1}^{-}\right)-\left(p_{2}^{-}+k_{2}^{-}\right)\right|\right) .
\end{aligned}
$$

In what follows, we approximate the cutoff functions by 1 , which is allowed to leading order for the range of cutoffs $g^{2} m^{2} \ll \Lambda^{2} \ll g m^{2}$ [2].

The " $\eta_{\mu} \eta_{\nu}$ " term is spin independent, it vanishes on shell, and it is at least one power of momenta higher than the leading spin-independent piece of the " $g_{\mu \nu}$ " term. As we explain later, it does not affect spin-spin and tensor interactions, but it may influence the spin-orbit.

In what follows we use Jacobi momenta:

$$
\begin{aligned}
& p_{i}^{+}=x_{i} P^{+} \quad, \quad p_{i}^{\perp}=\kappa_{i}^{\perp}, \\
& k_{i}^{+}=y_{i} P^{+}, \quad k_{i}^{\perp}=-\kappa_{i}^{\perp},
\end{aligned}
$$

where $y_{i}=1-x_{i}$; and we replace four-component spinors $u(p, \sigma), v(k, \lambda)$ with two- 
component spinors by substituting:

$$
u(p, \sigma)=\sqrt{\frac{2}{p^{+}}}\left(p^{+}+\beta m-\vec{\alpha}^{\perp} \cdot \vec{p}_{\perp}\right) \Lambda_{+}\left(\begin{array}{c}
\xi_{\sigma} \\
0
\end{array}\right),
$$

and similarly $v(k, \lambda)$. Here $\beta=\gamma^{0}, \vec{\alpha}=\gamma^{0} \vec{\gamma}$ are Dirac matrices, $\Lambda_{+}=\frac{1}{4} \gamma^{-} \gamma^{+}$is a projection operator, and $\xi_{\sigma}$ is a two-component spinor,

$$
\xi_{\uparrow}=\left(\begin{array}{c}
1 \\
0
\end{array}\right) \quad, \quad \xi_{\downarrow}=\left(\begin{array}{c}
0 \\
1
\end{array}\right) .
$$

From now on we will write the Hamiltonian as an operator which acts in the cross product space of these two-component spinors.

After the following change of variables, which defines $p_{z}$,

$$
x_{i}=\frac{\sqrt{{\overrightarrow{p_{i}}}^{2}+m^{2}}+p_{i z}}{2 \sqrt{{\overrightarrow{p_{i}}}^{2}+m^{2}}}, \quad y_{i}=\frac{\sqrt{{\overrightarrow{k_{i}}}^{2}+m^{2}}+k_{i z}}{2 \sqrt{{\overrightarrow{k_{i}}}^{2}+m^{2}}},
$$

where $\overrightarrow{k_{i}}=-\overrightarrow{p_{i}}$, and the three momentum in the center of mass frame is then $\vec{p} \equiv\left(\kappa^{\perp}, p_{z}\right)$, we take the nonrelativistic limit of the Hamiltonian.

The energy denominators become:

$$
\begin{aligned}
& q^{+} D_{1}=
\end{aligned}
$$

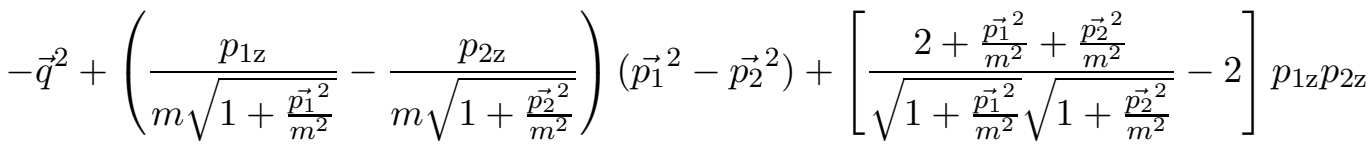

$$
\begin{aligned}
& \simeq-\vec{q}^{2} \text {, } \\
& q^{+} D_{2}=
\end{aligned}
$$

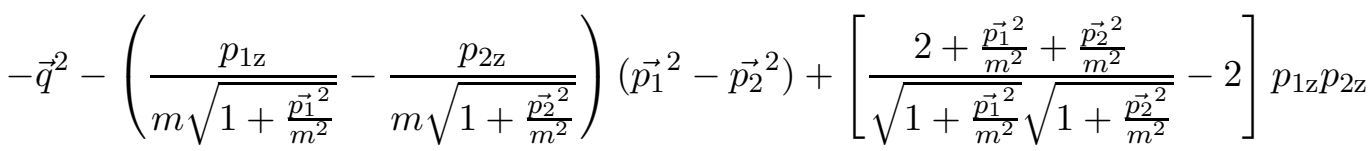

$$
\begin{aligned}
& \simeq-\vec{q}^{2},
\end{aligned}
$$


and the interaction Hamiltonian reduces to

$$
4 g^{2}(2 m)^{2}\left[\frac{1}{-\vec{q}^{2}}(1+d)\right]\left(v_{0}+v_{s p i n}\right)+v_{\eta_{\mu} \eta_{\nu}},
$$

where $v_{0}$ and $v_{\text {spin }}$ come from the $g_{\mu \nu}$ term in eqn. (4). $v_{0}$ is spin-independent and $v_{\text {spin }}$ depends on spins. $d$ denotes corrections from energy denominators that we discuss in the next section, together with the spin independent $v_{\eta_{\mu} \eta_{\nu}}$ that arises from the $\eta_{\mu} \eta_{\nu}$ term in eqn. (4). We have dropped an overall factor of $\sqrt{x_{1} x_{2}\left(1-x_{1}\right)\left(1-x_{2}\right)}$ in the Hamiltonian which is absorbed by a similar factor in the definition of the two-body wave function.

The corrections from energy denominators do not influence the discussion of spindependent structure, because they enter as an overall factor multiplying the entire $g_{\mu \nu}$ term. This will become clear later. To second order in powers of momenta,

$$
v_{0}=1+\frac{1}{4 m^{2}}\left(\vec{p}_{1}+\vec{p}_{2}\right)^{2}+\frac{1}{2 m^{2}} \vec{p}_{1} \cdot \vec{p}_{2}+\frac{1}{2 m^{2}} \vec{p}_{1}^{\perp} \cdot \vec{p}_{2}^{\perp}+\frac{3}{4 m^{2}}\left(\left(\overrightarrow{p_{1}}\right)_{z}^{2}+\left(\overrightarrow{p_{2}}\right)_{z}^{2}\right)
$$

and

$$
\begin{array}{r}
v_{\text {spin }}=-\frac{\mathrm{i}}{2 m}\left(\left(\vec{k}_{1}-\vec{k}_{2}\right) \times \vec{\sigma}_{a}\right)_{z}-\frac{\mathrm{i}}{2 m}\left(\left(\vec{p}_{1}-\vec{p}_{2}\right) \times \vec{\sigma}_{b}\right)_{z} \\
\quad+\frac{1}{4 m^{2}}\left(\left(\vec{k}_{1}-\vec{k}_{2}\right) \times \vec{\sigma}_{a}\right)_{\perp} \cdot\left(\left(\vec{p}_{1}-\vec{p}_{2}\right) \times \vec{\sigma}_{b}\right)_{\perp} \\
\quad+3 \frac{\mathrm{i}}{4 m^{2}}\left(\vec{k}_{2} \times \vec{k}_{1}\right) \cdot \vec{\sigma}_{a}+3 \frac{\mathrm{i}}{4 m^{2}}\left(\vec{p}_{2} \times \vec{p}_{1}\right) \cdot \vec{\sigma}_{b} \\
+\frac{\mathrm{i}}{4 m^{2}}\left(\vec{k}_{2} \times \vec{k}_{1}\right)_{z} \cdot\left(\vec{\sigma}_{a}\right)_{z}+\frac{\mathrm{i}}{4 m^{2}}\left(\vec{p}_{2} \times \vec{p}_{1}\right)_{z} \cdot\left(\vec{\sigma}_{b}\right)_{z} \\
+\frac{\mathrm{i}}{4 m^{2}}\left(\vec{k}_{1}\right)_{z} \cdot\left(\vec{k}_{1} \times \vec{\sigma}_{a}\right)_{z}-\frac{\mathrm{i}}{4 m^{2}}\left(\vec{k}_{2}\right)_{z} \cdot\left(\vec{k}_{2} \times \vec{\sigma}_{a}\right)_{z} \\
+\frac{\mathrm{i}}{4 m^{2}}\left(\vec{p}_{1}\right)_{z} \cdot\left(\vec{p}_{1} \times \vec{\sigma}_{b}\right)_{z}-\frac{\mathrm{i}}{4 m^{2}}\left(\vec{p}_{2}\right)_{z} \cdot\left(\vec{p}_{2} \times \vec{\sigma}_{b}\right)_{z} .
\end{array}
$$

We can immediately see that the first two terms in $v_{\text {spin }}$, which are linear in momentum, will lead to difficulties in bound state perturbation theory. In first-order bound-state 
perturbation theory they integrate to zero, but they enter at the second-order of boundstate perturbation theory, bringing the same power of momenta as the familiar term $\left(\vec{q} \times \vec{\sigma}_{a}\right)_{\perp} \cdot\left(\vec{q} \times \vec{\sigma}_{b}\right)_{\perp}$. So in order to obtain correct splitting of the ground state triplet and singlet states using this Hamiltonian, one has to sum second-order bound-state perturbation theory using all bound- and scattering electron-positron states [6]. Let us note that the remaining terms in (10) would give rise to part of the spin-orbit interactions.

The key to resolving this nuisance is to recognize that the spin-independent $v_{0}$ and the spin-dependent $v_{\text {spin }}$ are both multiplied by the same energy denominators. We try to find a unitary transformation which, applied to the spin-independent term, would generate terms cancelling the unwanted linear terms, and restoring rotational invariance in the $\left(\vec{q} \times \vec{\sigma}_{a}\right) \cdot\left(\vec{q} \times \vec{\sigma}_{b}\right)$ term.

Consider the following transformation:

$$
U_{\alpha}=1+\frac{\mathrm{i}}{2 m}\left(\overrightarrow{\mathcal{P}}_{\alpha} \times \vec{\sigma}_{\alpha}\right)_{z}-\frac{1}{2} \frac{\mathcal{P}_{\alpha}^{\perp 2}}{4 m^{2}}
$$

for each particle $\alpha$. This transformation is clearly unitary to second order in momenta, which is all we require here. For two particles $a$ and $b$ in the initial and final states,

$$
U_{\text {initial }}^{\dagger}=\left[1-\frac{\mathrm{i}}{2 m}\left(\vec{k}_{1} \times \vec{\sigma}_{a}\right)_{z}-\frac{1}{2} \frac{k_{1} \perp 2}{4 m^{2}}\right]\left[1-\frac{\mathrm{i}}{2 m}\left(\vec{p}_{1} \times \vec{\sigma}_{b}\right)_{z}-\frac{1}{2} \frac{p_{1}{ }^{\perp 2}}{4 m^{2}}\right] \text {, }
$$

and

$$
U_{\text {final }}=\left[1+\frac{\mathrm{i}}{2 m}\left(\vec{k}_{2} \times \vec{\sigma}_{a}\right)_{z}-\frac{1}{2} \frac{k_{2}{ }^{\perp 2}}{4 m^{2}}\right]\left[1+\frac{\mathrm{i}}{2 m}\left(\vec{p}_{2} \times \vec{\sigma}_{b}\right)_{z}-\frac{1}{2} \frac{p_{2}{ }^{\perp 2}}{4 m^{2}}\right]
$$

Then the Hamiltonian transforms as:

$$
H \rightarrow U_{f} H U_{i}^{\dagger}
$$


leading to new $v_{0}$ and $v_{\text {spin }}$ :

$$
\tilde{v}_{0}=1+\frac{1}{2 m^{2}}\left(\vec{p}_{1}+\vec{p}_{2}\right)^{2}+\frac{1}{2 m^{2}}\left({\overrightarrow{p_{1}}}^{2}+{\overrightarrow{p_{2}}}^{2}\right)
$$

to the leading order, and

$$
\begin{aligned}
\tilde{v}_{\text {spin }}= & -\frac{1}{4 m^{2}}\left(\vec{q} \times \vec{\sigma}_{a}\right) \cdot\left(\vec{q} \times \vec{\sigma}_{b}\right) \\
& +\frac{3 \mathrm{i}}{4 m^{2}}\left(\vec{k}_{2} \times \vec{k}_{1}\right) \cdot \vec{\sigma}_{a}+\frac{3 \mathrm{i}}{4 m^{2}}\left(\vec{p}_{2} \times \vec{p}_{1}\right) \cdot \vec{\sigma}_{b} \\
& -\frac{\mathrm{i}}{m^{2}}\left(\vec{p}_{2} \times \vec{p}_{1}\right) \cdot \vec{\sigma}_{b}-\frac{\mathrm{i}}{m^{2}}\left(\vec{k}_{2} \times \vec{k}_{1}\right) \cdot \vec{\sigma}_{a} \\
& +\frac{\mathrm{i}}{4 m^{2}}\left(\vec{p}_{1}\right)_{z} \cdot\left(\vec{p}_{1} \times \vec{\sigma}_{b}\right)_{z}-\frac{\mathrm{i}}{4 m^{2}}\left(\vec{p}_{2}\right)_{z} \cdot\left(\vec{p}_{2} \times \vec{\sigma}_{b}\right)_{z} \\
& +\frac{\mathrm{i}}{4 m^{2}}\left(\vec{k}_{1}\right)_{z} \cdot\left(\vec{k}_{1} \times \vec{\sigma}_{a}\right)_{z}-\frac{\mathrm{i}}{4 m^{2}}\left(\vec{k}_{2}\right)_{z} \cdot\left(\vec{k}_{2} \times \vec{\sigma}_{a}\right)_{z}
\end{aligned}
$$

The corrections from energy denominators (i.e. $d$ in eqn. (8)) do not affect spin-dependent interactions to this order. The term in the unitary transformation designed to remove $(\vec{q} \times \sigma)_{z}$ also removes $d(\vec{q} \times \sigma)_{z}$, because $d$ is an overall factor multiplying both $v_{0}$ and $v_{\text {spin }}$.

The rotationally noninvariant terms that do not mix initial and final state momenta (e.g., $\left.\frac{\mathrm{i}}{4 m^{2}}\left(\vec{p}_{1}\right)_{z} \cdot\left(\vec{p}_{1} \times \vec{\sigma}_{b}\right)_{z}\right)$ can be removed by adding terms of that form into the unitary transformation in the second order in momenta:

$$
U_{\alpha} \rightarrow U_{\alpha}+\frac{\mathrm{i}}{4 m^{2}}\left(\mathcal{P}_{\alpha}\right)_{z} \cdot\left(\overrightarrow{\mathcal{P}}_{\alpha} \times \vec{\sigma}_{\alpha}\right)_{z}
$$

The resultant spin-dependent interactions is:

$$
\begin{aligned}
\tilde{v}_{\text {spin }}= & -\frac{1}{4 m^{2}}\left(\vec{q} \times \vec{\sigma}_{a}\right) \cdot\left(\vec{q} \times \vec{\sigma}_{b}\right) \\
& +\frac{3 \mathrm{i}}{4 m^{2}}\left(\vec{k}_{2} \times \vec{k}_{1}\right) \cdot \vec{\sigma}_{a}+\frac{3 \mathrm{i}}{4 m^{2}}\left(\vec{p}_{2} \times \vec{p}_{1}\right) \cdot \vec{\sigma}_{b}
\end{aligned}
$$




$$
-\frac{\mathrm{i}}{m^{2}}\left(\vec{p}_{2} \times \vec{p}_{1}\right) \cdot \vec{\sigma}_{b}-\frac{\mathrm{i}}{m^{2}}\left(\vec{k}_{2} \times \vec{k}_{1}\right) \cdot \vec{\sigma}_{a}
$$

which is the familiar Breit-Fermi interaction.

The $\eta_{\mu} \eta_{\nu}$ term in eqn. (4) which we ignored so far is spin-independent and already one power of momenta higher than the leading spin-independent term in $v_{0}$. Therefore, to order two powers of momenta higher than the leading spin-independent term, it does not affect the spin-spin and tensor interactions. It may affect the spin-orbit interactions. But at least as far as the spin-spin structure, we can now diagonalize the new Hamiltonian using states that are related to the original states as

$$
|\tilde{\psi}\rangle=U|\psi\rangle
$$

It should be mentioned that the unitary transformation as presented here is a nextto-leading order expansion of the Melosh transformation [8]:

$$
\frac{m+x_{\alpha} M_{0}-\mathrm{i}\left(\mathcal{P}_{\alpha}^{\perp} \times \vec{\sigma}_{\alpha}\right)_{z}}{\sqrt{\left(m+x_{\alpha} M_{0}\right)^{2}+\mathcal{P}_{\alpha}^{\perp}}} .
$$

\section{Similarity transformation and fine structure}

In this section we consider corrections that arise due to the similarity transformation, i.e. the $\eta_{\mu} \eta_{\nu}$ term and the corrections due to energy denominators in the $g_{\mu \nu}$ term in (4). For completeness, we mention that the finite cutoffs also introduce corrections, the size of which depends on the specific choice of $\Lambda$ [2].

In the previous considerations we omitted corrections due to energy denominators in the $g_{\mu \nu}$ term in (4), or $d$ in eqn. (8). These corrections do not affect the spin-dependent 
terms, but they do produce a spin-independent correction:

$$
4 g^{2}(2 m)^{2}\left[\frac{d}{\vec{q}^{2}}\right]=4 g^{2}(2 m)^{2} \frac{1}{\vec{q}^{2}}\left[\frac{\left|q_{z}\right|\left|\vec{q} \cdot\left(\overrightarrow{p_{1}}+\overrightarrow{p_{2}}\right)\right|}{m \vec{q}^{2}}\right]
$$

where we have dropped the omnipresent $\sqrt{x_{1} x_{2}\left(1-x_{1}\right)\left(1-x_{2}\right)}$ as before. Similarly, any corrections of this term due to finite cutoff do not affect spin-dependent interactions.

We now address the $v_{\eta_{\mu} \eta_{\nu}}$ term, and its effect on the spin-orbit interaction. Dropping the omnipresent $\sqrt{x_{1} x_{2}\left(1-x_{1}\right)\left(1-x_{2}\right)}$, the $\eta_{\mu} \eta_{\nu}$ term gives

$$
4 g^{2} \frac{1}{2\left(x_{1}-x_{2}\right)^{2}}\left[\frac{\theta\left(\left|D_{1}\right|-\left|D_{2}\right|\right)\left(q^{+} D_{1}-q^{+} D_{2}\right)}{q^{+} D_{1}}+\frac{\theta\left(\left|D_{2}\right|-\left|D_{1}\right|\right)\left(q^{+} D_{2}-q^{+} D_{1}\right)}{q^{+} D_{2}}\right] .
$$

To the lowest order in momenta this equals (for details see appendix):

$$
v_{\eta_{\mu} \eta_{\nu}}=4 g^{2}(2 m)^{2} \frac{1}{\vec{q}^{2}}\left[\frac{\left|q_{z}\right|\left|\vec{q} \cdot\left(\overrightarrow{p_{1}}+\overrightarrow{p_{2}}\right)\right|}{m q_{z}^{2}}\right] .
$$

The unitary transformation (14) applied to this term produces Đ:

$$
4 g^{2}(2 m)^{2} \frac{1}{\vec{q}^{2}}\left[\frac{\left|q_{z}\right|\left|\vec{q} \cdot\left(\frac{\overrightarrow{p_{1}}}{m}+\frac{\overrightarrow{p_{2}}}{m}\right)\right|}{q_{z}^{2}}\right]\left[1-\frac{\mathrm{i}}{2 m}\left(\vec{q} \times \vec{\sigma}_{a}\right)_{z}+\frac{\mathrm{i}}{2 m}\left(\vec{q} \times \vec{\sigma}_{b}\right)_{z}\right] .
$$

All of these corrections are nonanalytic. This is a consequence of using a nonanalytic cutoff function in the similarity transformation. If the nonanalytic spin-dependent corrections do not vanish, a simple the angular momentum operator does not emerge even in the nonrelativistic limit. Fortunately, these spin-dependent terms integrate to zero in the first order bound state perturbation theory, since they are odd under parity. Terms

\footnotetext{
${ }^{1}$ This term can affect spin-orbit splittings even if one does not use the unitary transformation to rotate the spins. In that case, there would be correction in second order bound state perturbation theory, arising from the product of (21) with the first two terms in $v_{\text {spin }}$ (see eqn. (10)).
} 
that appear at higher orders must be paired with other terms from second order bound state perturbation theory and with terms from higher order similarity transformation. Corrections which arise due to finite value of cutoff $\Lambda$ do not influence the lowest order of spin-dependent interactions for the same reasons.

\section{Conclusions}

We have studied the part of the effective QED Hamiltonian that does not change particle number. We have shown that the light-front spin-dependent interactions reduce to the familiar Breit-Fermi interactions. This can be achieved by a simple unitary transformation corresponding to a change of spinor basis. As a consequence of sharp cutoff functions in the similarity transformation, there are nonanalytic corrections. These nonanalytic corrections produce spin-independent corrections at $\mathcal{O}\left(\alpha^{3}\right)$, but they do not affect the spin-dependent splittings at order $\mathcal{O}\left(\alpha^{4}\right)$. Photon exchange below the cutoff is needed to remove the corrections at order $\mathcal{O}\left(\alpha^{3}\right)$.

Our primary motivation for restricting the study to the part of Hamiltonian which does not change the particle number was QCD. The approach suggested by Wilson et al [1] builds on suppressing the exchange of low energy gluons by introducing a gluon mass. This makes higher Fock states less important.

As far as aspects discussed here, in QCD questions about rotational symmetry become more complicated, because the finite size of $\Lambda$ comes to play. It is straightforward to show that the procedure we outlined here does not completely restore manifest rotational 
invariance in the spin-spin interaction in QCD. It is still useful, however, because it helps to separate violations of manifest rotational symmetry caused by using light-front spinors from the violations due to the cutoff [5].

\section{Acknowledgements}

This work was supported by the National Science Foundation under grant PHY-9409042. M.B. would like to thank R. Furnstahl for his valuable comments that helped me to improve this manuscript, and S. Głazek for discussions.

\section{Appendix: $\eta_{\mu} \eta_{\nu}$ term}

Let us concentrate on the expression in the square brackets in eqn. (20). From eqn. (6) for the energy denominators one can see that the energy denominators have form:

$$
\begin{aligned}
& q^{+} D_{1}=a+X \\
& q^{+} D_{2}=a-X,
\end{aligned}
$$

where

$$
X=\left(\frac{p_{1 \mathrm{z}}}{m \sqrt{1+\frac{\vec{p}_{1}^{2}}{m^{2}}}}-\frac{p_{2 \mathrm{z}}}{m \sqrt{1+\frac{\vec{p}_{2}^{2}}{m^{2}}}}\right)\left({\overrightarrow{p_{1}}}^{2}-{\overrightarrow{p_{2}}}^{2}\right)
$$

and

$$
a=-\vec{q}^{2}+\mathcal{O}\left(p^{6}\right)
$$


The difference between the energy denominators is

$$
q^{+} D_{1}-q^{+} D_{2}=2 X
$$

Theta functions can be expressed as

$$
\begin{array}{r}
\theta\left(\left|D_{1}\right|-\left|D_{2}\right|\right)=\theta(|a+X|-|a-X|)=\theta(a X) \\
\theta\left(\left|D_{2}\right|-\left|D_{1}\right|\right)=\theta(|a-X|-|a+X|)=\theta(-a X) .
\end{array}
$$

Using these expressions, to the lowest nonvanishing order:

$$
\begin{aligned}
& {\left[\frac{\theta\left(\left|D_{1}\right|-\left|D_{2}\right|\right)\left(q^{+} D_{1}-q^{+} D_{2}\right)}{q^{+} D_{1}}+\frac{\theta\left(\left|D_{2}\right|-\left|D_{1}\right|\right)\left(q^{+} D_{2}-q^{+} D_{1}\right)}{q^{+} D_{2}}\right]} \\
& \quad=2\left[\frac{X \theta(a X)}{a+X}-\frac{X \theta(-a X)}{a-X}\right] \simeq 2\left|\frac{X}{a}\right|+\mathcal{O}\left(\frac{X^{2}}{a^{2}}\right) .
\end{aligned}
$$

It is easy to show that even if the cutoff $\Lambda$ is kept in place, the nonanalytic corrections are still present.

\section{References}

[1] K. G. Wilson, T. S. Walhout, A. Harindranath, Wei-Min Zhang, R. J. Perry and S.D. Głazek, Phys. Rev. D 49, 6720 (1994), hep-th/9401153.

[2] R. J. Perry, in Proceedings of Hadrons 94, edited by V. Herscovitz and C. Vasconcellos (World Scientific, Singapore, 1995), hep-th/9407056.

[3] St. D. Głazek and K. G. Wilson, Phys. Rev. D 48, 5863 (1993); ibid. 49, 4214 (1994). Similar flow equations for Hamiltonians were proposed by F. Wegner, Ann. Physik 3, 77 (1994). 
[4] R. J. Perry and K. G. Wilson, Nuc. Phys. B 403, 587 (1993); R. J. Perry, Ann. Phys. 232, 116 (1994), hep-th/9402015.

[5] M. Brisudova and R. Perry, hep-ph/9511443, to appear in Phys. Rev. D(1996).

[6] B. Jones, R. Perry, S. Głazek, in preparation.

[7] M. Kaluza and H.-J. Pirner, Phys. Rev. D 47, 1620 (1992).

[8] H. J. Melosh, Phys. Rev. D 9, 1095 (1974). 